\title{
Augmentative and alternative communication on tablet to help persons with severe disabilities
}

\author{
Daniela Grigis Marco Lazzari \\ University of Bergamo \\ Department of Human and Social Sciences \\ Piazzale Sant'Agostino 2, Bergamo, Italy \\ marco.lazzari@unibg.it
}

\begin{abstract}
In this paper we present an experience of the application of augmentative and alternative communication (AAC) with four persons with severe language (and motor) impairments treated at an adult day centre for people with disabilities. Two apps on Android tablets were tried with one subject who had negatively experienced traditional communication boards in the past and with three subjects who had never been considered before for AAC intervention. After a sixmonth trial we achieved encouraging results: the ease of use of the proposed instruments enabled the persons involved in the experimentation to improve their communication capabilities, to better express their choices and feelings, and to potentially extend their communication circles. This experience testifies that AAC can be successfully applied to severe disabilities and encourages research in this direction.
\end{abstract}

\section{Author Keywords}

Augmentative and alternative communication; severe disabilities; language impairments; tablet; Android O.S.; speech generating devices; picture communication symbols (PCS); user centered design; person centered design.

\section{ACM Classification Keywords}

C.5.3. Microcomputers: Portable devices. H.1.2. User/Machine Systems: Human factors. H.5.2. User Interfaces: User-centered design. H.5.m. Information interfaces and presentation (e.g., HCI): Miscellaneous. K.4.2. Computers and society, Social Issues: Assistive technologies for persons with disabilities.

\section{General Terms}

Human Factors; Design.

\section{INTRODUCTION}

Augmentative and alternative communication (AAC) is a broad term used to address communication methods and techniques that help people with language impairments so that they can supplement or replace natural speech or writing and understand or produce spoken or written language.

Permission to make digital or hard copies of all or part of this work for personal or classroom use is granted without fee provided that copies are not made or distributed for profit or commercial advantage and that copies bear this notice and the full citation on the first page. Copyrights for components of this work owned by others than the author(s) must be honored. Abstracting with credit is permitted. To copy otherwise, or republish, to post on servers or to redistribute to lists, requires prior specific permission and/or a fee. Request permissions from Permissions@acm.org.

CHItaly '13, September 16 - 20 2013, Trento, Italy

Copyright is held by the owner/author(s). Publication rights licensed to

ACM. ACM 978-1-4503-2061-0/13/09...\$15.00.

http://dx.doi.org/10.1145/2499149.2499175.
AAC solutions can be regarded as technological answers to the request for research and development promoted by the United Nations Convention on the Rights of Persons with Disabilities [12], which provides (Art. 4) that "States Parties $[\ldots]$ undertake or promote research and development of, and to promote the availability and use of new technologies, including information and communications technologies, mobility aids, devices and assistive technologies, suitable for persons with disabilities". In the same document, the section significantly titled Living Independently and Being Included in the Community (Art. 19) provides that the States Parties "shall take effective and appropriate measures to facilitate $[\ldots]$ their [of the persons with disabilities] full inclusion and participation in the community"; and later (Art. 21), that they "shall take all appropriate measures to ensure that persons with disabilities can exercise the right to freedom of expression and opinion, including the freedom to seek, receive and impart information and ideas on an equal basis with others".

AAC may also fulfill the requests of the Communication Bill of Rights contained in the Guidelines for Meeting the Communication Needs of Persons with Severe Disabilities [10], which aims to provide people with severe impairments with the rights "to express personal preferences, or feelings" (Art. 1), "to be offered choices and alternatives" (Art. 2), " to request, and be given, attention from and interaction with another person" (Art. 4), and "to participate as full communication partners with other people" (Art. 9) - the same concept is proposed in similar terms by the Commission of the European Communities with its initiative on eInclusion, which says that the aim of the campaign "is to enable every person who so wishes to fully participate in the information society, despite individual or social disadvantages" [3].

AAC is currently adopted to treat several congenital and acquired conditions such as cerebral palsy, intellectual disability, autism, verbal dyspraxia, locked-in syndrome, amyotrophic lateral sclerosis, Parkinson's disease, multiple sclerosis, dementia, aphasia and traumatic brain injuries.

AAC methods can be of two types:

- Unaided communication: This does not require any instrument, but only the use of the body, such as with facial expressions, gestures, or sign languages. They can have a 
great generative potential, but require fine motricity, which is not the case with the subjects of the present trial.

- Aided communication: This happens when inclusive technologies are used.

Aided technologies may be divided into low-, medium- and high-tech. Low-tech solutions are those provided by aids that do not need electricity, such as the so-called communication boards, which allow users to select letters, words, or symbols, depending on their physical and cognitive limitations. Several symbolic systems, which are also used at the medium- and high-tech level, have been developed, such as Blissymbolics (see Figure 1) or the Picture Communication Symbols (PCS; see Figure 2).

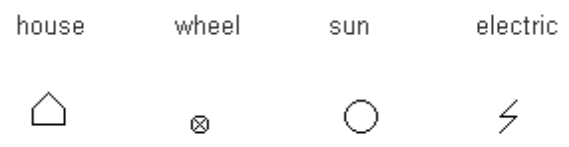

Figure 1. Bliss symbols (CBlissymbolics Communication International).

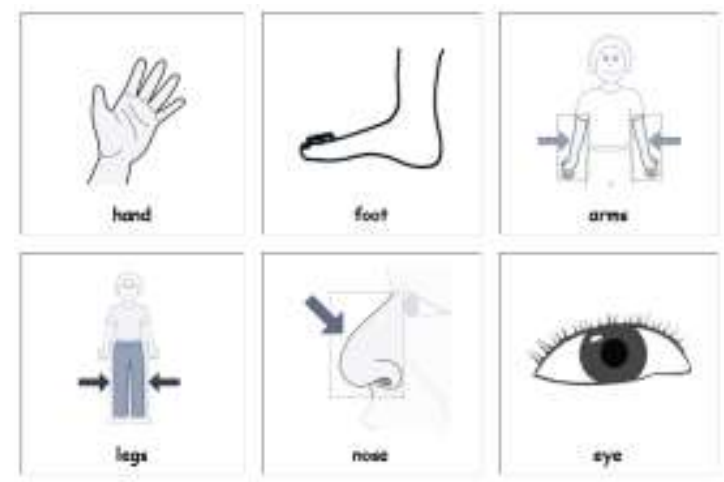

Figure 2. Picture communication symbols (CDo2Learn).

Medium-tech solutions are those provided by communication-oriented electronic devices that are not required to be based with nor connected to a general purpose computer, such as the Vocal Output Aids (VOCA), which are communication boards made of buttons, each of which is connected to a symbol or a pre-recorded message: by pressing the button, the speech synthesizer reads the corresponding word or phrase. High-tech solutions are those exploiting and integrating computers, multimedia interfaces, speechgeneration, and multimedia acquisition from external sources.

The current state of information technology has led in recent years to a key development in the application of computers to the AAC: the information technology evolution, through the progressive miniaturization of both the size and cost of devices and the advent of mobile computing, has provided us with computers that have ceased to be as they were in the early decades of the history of computing - that is calculation tools equipped with communication devices to exchange calculation results with the outside world - and they have become primarily communication tools equipped with computing devices to process information flows to and from the outside world.

Miniaturized components have generated two key properties of modern devices: portability and transparency. Both have a great impact on possible uses by persons with speech impairments: portability allows mobile use, so that the same device can be exploited in different ecological microsystems [2] (e.g. family, day care center) and also to foster mesosytemic interactions (e.g. between the two); transparency means here the property of small size processing systems, so that their reduced size decreases the psychological acceptance threshold for assistive devices.

AAC can especially benefit from tablets, which outperform dedicated first generation AAC devices in terms of screen resolution, pixel density, battery life, weight and price [5].

The technical literature on this matter is still poor, but reports already exist about the viability of iOS based technological aids for individuals with developmental disabilities [8], the appropriateness of apps for persons with complex communication needs (who can use them for a variety of communication purposes) [1], the opportunities given to students with special education needs by mobile learning solutions to perform activities that were not accessible to them in the past [6], and about the promising potential effect that Android based devices could have on AAC [7]. As we shall see in the following, we agree that technology is crucial for addressing speech impairments, but much more so is the ability to exploit it [4]; this requires a sound methodological and pedagogical point of view, as well as care, technical competence, and a person-centered approach to adapting customizable software to the specific and particular needs of each person that is going to use the AAC digital solution.

\section{AN ACTION RESEARCH ON THE APPLICATION OF AAC TO SEVERE DISABILITIES}

In this chapter we shall synthetically describe an attempt to apply AAC using two apps running on a tablet with four adults with severe language (and motor) impairments being treated at a day care center for people with disabilities. This was done through a participation action research [9] performed over a time span of six months, from September 2012 to February 2013. The four subjects were supported by an assistant, who progressively trained them in the use of a tablet and built appropriate personalized symbol tables to help them in basic communicative interactions, such as choosing the activities to be undertaken at the center or their favorite dish from the lunch menu. A daily journal was filled in to track the steps and results of the activity. In the following, we propose and discuss a synthesis of the main evidences recorded and reflexively revised.

\section{Choosing the candidates}

Within the scientific community there is no consensus about the existence of candidacy criteria for AAC interven- 
tions [11]; for our part, we decided to select for our program four persons who were able to manifest clear communication intentionality and to rather intelligibly express the difference between yes and no by means of either verbal or non verbal communication (or both). Among them, one had already unsuccessfully been exposed to traditional AAC methods at school, whereas the other three had never been considered before for AAC interventions. None of them had acquired reading ability. The number of subjects for the trial was limited to four because a single research assistant was available for the experimentation.

\section{Aims}

At the beginning of the action research we defined some objectives to strive for in using the device:

- to motivate use of the device for communicating

- to use the device in different contexts

- to use the device for expressing primary needs

- to properly use the device with the educators

- to increase motivation for communicating with other people than the educators at the care center

- to improve eye-hand coordination

- to create or increase awareness of participation in everyday life.

\section{Choosing hardware and operating system}

For reasons related to costs we decided to opt for a non-iOS platform, which seemed a more reasonable proposition to the families for possible future purchase. The Android operating system was selected, due to its diffusion and the number of potential free apps available on the market.

Some preliminary tests were conducted on several tablets; after a first round, screens under the 10" were discarded, because they did not seem suitable for people with limited visual acuity and/or reduced fine motor skills; at the end, a Samsung Galaxy Tab 2 10.1 (1 GHz Dual Core processor, 1 GB RAM, 10.1" screen 1280×800 px) with Android OS version 4.0.4 "Ice Cream Sandwich" was chosen. Some experiments were also made on a smaller Samsung Galaxy Tab 27.

\section{Choosing apps}

Many apps were downloaded from the multimedia market Google Play, were tried and evaluated with reference to the project needs, that is, the requirement for:

- free license

- high customizability

- user friendliness, simple and clear interface structure

- Italian or English interface

- integration of text-to-speech facilities.
The following apps were checked against those requirements and discarded: Niki Talk (version 1.3.3), Logopedia (v. 1.8), Plaphoons (v. 2.0.2), Alexicom AAC (v. 1.1.1), PictoDroid Lite (v. 2.1.5), and AAC speech (v. 1.0 beta). Eventually, we decided to use two apps: JABtalk (v. 4.2.7) and AAC Talking Tabs (v. 1.1.4.4). Both allow the management of categories, insertion of pictures, and generation of vocal output; the former provides a tree structure for organizing symbols and pictures that is simply navigated step by step by the user; the latter consents to building phrases by moving from one picture to another.

\section{Co-designing a personalized communication table with the users}

With each user we co-designed a personalized communication table, through a very long and time expensive process: initially we had to get the users familiar with the device and the interaction modalities; then, photographs were taken for any situation, object, or person had to become part of the vocabulary of each user (PCS were preferred when more effective); eventually, users were instructed on how to use their tables to answer requests and express their desires or feelings.

Approaching the persons involved in the experimentation was rather challenging, since some of them are often oppositional and hostile toward authority figures, suffer from lack of concentration or have a limited attention span. Therefore, the task of co-designing was really hard and the development phases had to be strategically interleaved with entertainment moments and reinforcements.

At the end of the development and training phase, all the users had shown interest in the new form of communication. They had acquired communication skills that allowed them to express their preferences, for instance for choosing what to eat or drink (one of them is now regularly choosing espresso coffee after lunch, whereas for more than a year she was given barley coffee because nurses were convinced that she would prefer that kind of drink), and they exploited the possibility of creating conversational acts beyond the question/answer scheme. As an example, the following discussion took place: "May I have one more biscuit? No, you can't. So, I shall eat them at home". It is evident that this kind of interaction was hardly achievable previously with the persons who had only mastered the yes/no answer.

\section{DISCUSSION}

The main goal of our exploratory action research was to verify whether the tablet devices with their apps were appropriate to facilitate the communication of people with severe disabilities, such as those treated at the center where the trial took place. The results of the work are more than satisfactory.

With reference to the use of tablets, these devices have proved easy and intuitive to use, even by people who are both physically and cognitively very compromised, given 
the simplicity of the touchscreen interaction approach. The simple imitation of operator's gestures allowed the subjects involved in the research to easily interact with the device from the first attempts, despite their serious motor impairments, and the finger-driven interface was accepted by them, according to their different abilities. The ease of use of the interactive platform allowed the persons involved in our experiment to greatly extend their communication opportunities. With reference to the Communication Bill of Rights [10], they were given the opportunity to better enjoy their right to "request desired objects, actions, events, and persons, and to express personal preferences, or feelings", and more generally to be offered alternatives to choose from (Art. 2).

The speech synthesis capability helped them to understand and remember the meaning of images and symbols that were clicked, allowing an immediate check of the messages built through the tablet. At the same time, it made it possible to establish communication relations with different people, without the need to explain the method, in a more transparent way than with respect to communication acts based on ordinary AAC boards. With reference to the Communication Bill of Rights, this implements the right to interact with another person, to request and be given attention, and to participate as full communication partners with other people (Art. $4 \& 9$ ).

The scope for customizing the programs chosen for the experimentation and the flexibility of their graphical interface were of paramount importance in optimizing their use with different subjects and in adapting them to the needs of each person.

The symbolic PCS method proved to be easy to understand and communicatively efficient. Most users intuitively understood the meaning of most concrete symbols, while for the abstract ones a simple explanation was enough to facilitate comprehension and use. Images or personal photographs used as an alternative to symbols proved to be an effective way of expressing specific concepts or communicating when a person seemed to better understand pictures rather than PCS.

Moreover, we found a significant increase in the intentionality of communication, which was encouraged by the effectiveness of the system and the friendliness of the interface. In the terms of the Communication Bill of Rights it has resulted in the opportunity to express personal preferences, or feelings (Art. 1).

To summarize, our findings show how even people who are severely compromised, who are often exempted from traditional AAC solutions, may communicate with adequately personalized products. This requires one-off customization and great effort in order to be performed, and it can be regarded as a form of inclusive person-centered co-design aimed at acknowledging and enhancing the inherent dignity of the person being involved in the communication project.

\section{ACKNOWLEDGMENTS}

We are grateful to the four people with whom we worked for the extraordinarily enriching experience that they made us live and share with them. We thank the Day Care Center of Piario that hosted our project.

\section{REFERENCES}

1. Alliano, A., Herriger, K., Koutsoftas, A.D., and Bartolotta, T.E. A review of $21 \mathrm{iPad}$ applications for augmentative and alternative communication purposes. Perspectives on Augmentative and Alternative Communication, 21, 2 (2012), 60-71.

2. Bronfenbrenner, U. The ecology of human development. Harvard University Press, Cambridge, MA, USA, 1979.

3. Commission of the European Communities. European i2010 initiative on e-Inclusion. 2007.

http://ec.europa.eu/information_society/activities/einclu sion/docs/i2010_initiative/comm_native_com_2007_06 94_f_en_acte.pdf

4. Cook, A. It's not about the technology, or is it? Realizing AAC through hard and soft technologies. Perspectives on Augmentative and Alternative Communication, 20, 2 (2011), 64-68.

5. Dolic, J., Pibernik, J., and Bota, J. Evaluation of mainstream tablet devices for symbol based AAC communication. In Proc. KES-AMSTA 2012, Springer, Berlin, Germany, 2012.

6. Fernández-López, Á., Rodríguez-Fórtiz, M.J., Rodríguez-Almendros, M.L., and Martínez-Segura, M.J. Mobile learning technology based on iOS devices to support students with special education needs. Computers \& Education, 61 (2013), 77-90.

7. Higginbotham, J. The future of the Android operating system for augmentative and alternative communication. Perspectives on Augmentative and Alternative Communication, 20, 2 (2011), 52-56.

8. Kagoara, D.M. et al. Using iPods $(\subset$ and iPads $($ in teaching programs for individuals with developmental disabilities: A systematic review. Research in Developmental Disabilities, 34, 2 (2013), 147-156.

9. McIntyre, A. Participatory action research. Sage Publications, Thousand Oaks, CA, USA, 2008.

10. National Joint Committee for the Communication Needs of Persons with Severe Disabilities. Guidelines for meeting the communication needs of persons with severe disabilities. 1992. http://www.asha.org/docs/html/GL1992-00201.html

11. Romski, M.A., and Sevcik, R. (2005). Augmentative communication and early intervention: Myths and realities. Infants \& Young Children, 18, 3, 174-185.

12. United Nations. Convention on the rights of persons with disabilities. UN, New York, NY, USA, 2006. 